\title{
Electron localization into a bound spin polaron in the quasi-one-dimensional $S=\frac{1}{2}$ antiferromagnet $\mathrm{LiCu}_{2} \mathrm{O}_{2}$
}

\author{
Vyacheslav G. Storchak, ${ }^{1, *}$ Jess H. Brewer, ${ }^{2}$ Donald J. Arseneau, ${ }^{3}$ Scott L. Stubbs, ${ }^{2}$ Oleg E. Parfenov, ${ }^{1}$ \\ Dmitry G. Eshchenko, ${ }^{4,5}$ and Alexander A. Bush ${ }^{6}$ \\ ${ }^{1}$ Russian Research Centre "Kurchatov Institute," Kurchatov Square 1, Moscow 123182, Russia \\ ${ }^{2}$ Department of Physics and Astronomy, University of British Columbia, Vancouver, British Columbia, Canada V6T 1 Z1 \\ ${ }^{3}$ TRIUMF, 4004 Wesbrook Mall, Vancouver, British Columbia, Canada V6T $2 A 3$ \\ ${ }^{4}$ Physik-Institut, Universität Zürich, CH-8057 Zürich, Switzerland \\ ${ }^{5} \mathrm{SuS}$, Paul Scherrer Institute, CH-5232 Villigen, Switzerland \\ ${ }^{6}$ Institute for Radiotechnics, Electronics, and Automation, Moscow 117464, Russia
}

(Received 20 April 2009; published 12 June 2009)

\begin{abstract}
Muon spin rotation experiments in zero magnetic field and magnetization measurements have been carried out in a single crystal of spin $S=1 / 2$ double chain cuprate $\mathrm{LiCu}_{2} \mathrm{O}_{2}$ over a temperature range of $2-300 \mathrm{~K}$. In the antiferromagnetic state we find a bound state of an electron around the muon - the magnetic polaron - with the electron wave function confined within $R=0.55 \pm 0.05 \mathrm{~nm}$. Electron localization in this form persists up to $31 \mathrm{~K}$, well above $T_{N}=24.7 \mathrm{~K}$.
\end{abstract}

DOI: 10.1103/PhysRevB.79.220406

PACS number(s): 71.38.Ht, 75.50.Ee, 74.72.Jt, 76.75.+i

In many materials, interaction of conduction electrons with the medium is strong enough to qualitatively change the state of the carrier. The general picture depends critically on the adiabaticity of its interaction with excitations of the environment. In systems with large electronic bandwidth $E_{B}$ such that $E_{B} \gg \omega$ (the characteristic phonon frequency), carrier transport can be accurately described in the framework of the adiabatic approximation (Fermi-liquid theory). In narrower band systems, carrier motion becomes nonadiabatic as it is followed "instantaneously" by phonon modes and is best thought of as a quasiparticle-a polaron ${ }^{1}$ — whose mobility is drastically decreased [the lattice polaron (LP)].

In a magnetic material, the exchange interaction between conduction electrons and localized spins creates an additional channel for electron localization. In metals, where the exchange coupling parameter $I$ is small compared to the Fermi energy $E_{F}$, the effects of the exchange are modest, as illustrated by the Kondo effect. In magnetic semiconductors (MSs), however, the exchange coupling is strong ${ }^{2}$ while the kinetic energy of the free carriers is small as they occupy energy levels close to the bottom of the conduction band. Then the opposite limit $\left(E_{F}<I\right)$ may be realized, dramatically influencing the electron state. In particular, the exchange interaction between the free carrier and magnetic ions may cause electron localization into a ferromagnetic (FM) "droplet" on the scale of the lattice spacing in a paramagnetic (PM) or antiferromagnetic (AF) "sea., 2,3" The charge carrier coupled to its immediate FM environment together behave as one entity, referred to as a magnetic polaron (MP), with a large composite spin $\mathcal{S}$.

The MP concept now forms the basis for numerous studies of MS and related materials. In particular, the remarkable transport and optical properties of these materials are discussed in the framework of MP formation. ${ }^{4}$ Recent direct observations of the magnetic polaron in the PM phase of SmS (Ref. 5), EuS, EuO, EuSe, and $\mathrm{CdCr}_{2} \mathrm{Se}_{4}$ (Ref. 6) have revealed electron localization within a FM cluster about 0.6 $\mathrm{nm}$ across (i.e., the first coordination sphere) due to ex- change interaction with $\mathrm{Eu}(\mathrm{Cr})$ magnetic moments. In EuS, the composite spin of the MP thus formed is determined ${ }^{5}$ to be $\mathcal{S}=36 \pm 4$.

In ferromagnetic MS, below the transition temperature $T_{c}$ the lattice spins are already aligned; the exchange coupling of the carrier with these spins is then of no particular significance. In fact, as the magnetization develops toward low temperature, the electron avoids localization at temperature higher than $T_{c}$. In contrast, in AF semiconductors the magnetization decreases as $T \rightarrow T_{N}$ from above. The carrier may then be localized even at zero temperature. ${ }^{3,7}$

The scaling approach to both lattice ${ }^{8}$ and magnetic ${ }^{9}$ polarons has shown that the conditions for polaron formation depend critically on the dimensionality of the system. In a three-dimensional (3D) system, the LP is separated by an energy barrier from the free carrier state while in twodimensional (2D) and one-dimensional (1D) systems the LP forms without any energy barrier. ${ }^{10}$ The MP is most stable in a 1D AF; in a 3D lattice the MP is stable only when it is bound to some center [the so-called bound magnetic polaron (BMP)]. ${ }^{11,12}$ Thus polaron formation is encouraged by the general tendency of the electronic bandwidth $E_{B}$ to be narrower as the dimensionality gets lower. ${ }^{2}$

Studies of polaron formation and transport in lowdimensional systems are also motivated by considerations of charge transport within the $\mathrm{CuO}_{2}$ planes in superconducting cuprates. In most of these materials, holes created by substituent doping or variable oxygenation cause a transition from an insulating AF parent compound to a "strange metal" that becomes superconducting below $T_{c}$. Their discovery prompted early theoretical attempts to explain the origin of high- $T_{c}$ superconductivity in terms of magnetic interactions. ${ }^{13-15}$ Some of the proposed models involve local magnetic order around a localized carrier. (For a recent review, see Ref. 16 and references therein.) A hole may be localized by the combined effects of charge-phonon interaction, causing local distortion of the lattice (LP-like) ${ }^{17}$ and spin-charge interaction leading to a local distortion of the AF order (MP-like). ${ }^{18}$ 
The general problem of a charge carrier in an AF lattice has been studied within the Hubbard or $t-J$ models. This problem is intimately connected to formation of the spin polaron (SP): at low temperature, the competition between the kinetic energy of the hole $(\sim t)$ and the (super)exchange interaction $(\sim J)$ results in localization of the hole in the distorted AF environment. ${ }^{19}$ A numerical solution ${ }^{20}$ shows that the MP exists if $(t / J)$ is larger than some critical value $(t / J)_{c} \sim 50$. If $(t / J)<(t / J)_{c}$, the so-called "string SP" is believed to be a more favorable excitation. ${ }^{19}$

One-dimensional $S=1 / 2$ AF compounds based on copperoxide "chains" or "ladders" form a family related to the high- $T_{c}$ cuprates. Both transport properties and electronic structure of these materials also involve considerations based on the Hubbard model. Compounds with coupled $S=1 / 2$ chains are particularly interesting as they represent a structure intermediate between 1D and 2D systems. Within this family, long-range AF order has been found in systems with zigzag chain structure $\left(\mathrm{SrCuO}_{2}\right)$ (Ref. 21) and with weak $\left(\mathrm{Sr}_{2} \mathrm{CuO}_{3}\right.$ and $\left.\mathrm{Ca}_{2} \mathrm{CuO}_{3}\right)$ (Ref. 22) or comparatively strong $\left(\mathrm{LiCu}_{2} \mathrm{O}_{2}\right)$ interchain exchange interaction. The latter has recently received special attention ${ }^{23-28}$ because of expected quantum effects. In this Rapid Communication we report observation of BMP formation in $\mathrm{LiCu}_{2} \mathrm{O}_{2}$.

$\mathrm{LiCu}_{2} \mathrm{O}_{2}$ has a layered orthorhombic crystal structure (space group Pnma). It is a mixed-valent compound whose unit cell contains equal numbers of $\mathrm{Cu}^{1+}$ and $\mathrm{Cu}^{2+}$ ions. ${ }^{24,25}$ Its magnetism is due to two linear $\mathrm{Cu}^{2+}$ chains that run along the $\hat{b}$ axis and form a zigzag ladderlike structure, with these ladders being effectively isolated from each other by chains of $\mathrm{Li}$ and $\mathrm{O}$ ions in the $\hat{a}$ direction and the layers of nonmagnetic $\mathrm{Cu}^{1+}$ ions along the $\hat{c}$ direction. ${ }^{23,25}$ Competition between a combination of nearest-neighbor FM and next nearest-neighbor $\mathrm{AF}$ interactions and an additional longrange AF coupling causes magnetic frustration. As a result of this frustration, a spiral (helicoidal) magnetic structure is realized below $T_{N} \sim 24 \mathrm{~K}^{23}$ Magnetization, ${ }^{25,27,28} \mathrm{ESR},{ }^{25}$ specific-heat, ${ }^{24,25}$ muon spin rotation $\left(\mu^{+} \mathrm{SR}\right),{ }^{24}$ neutron, ${ }^{23,28}$ dielectric,,$^{27,28}$ angle resolved photoemission spectroscopy, and optical ${ }^{26}$ studies all reveal that long-range AF order sets in at $T_{N}$ between 23 and $25 \mathrm{~K}$. Note, however, that previous experiments ${ }^{23-28}$ were all carried out in multidomain (twinned in the $a b$ plane) single crystals of $\mathrm{LiCu}_{2} \mathrm{O}_{2}$.

For the current measurements a single crystal of $\mathrm{LiCu}_{2} \mathrm{O}_{2}$ was grown in an alundum crucible using a self-flux technique. X-ray diffraction measurements at room temperature (using monochromatic $\mathrm{Cu} K \beta$ radiation) have revealed no traces of any parasitic phases. The sample exhibits no $90^{\circ}$ twinning within the $a b$ plane: a single-domain pattern is clearly seen in Fig. 1. Crystalline mosaicity does not exceed $0.3^{\circ}$ (full width at half height). The lattice parameters $[a=0.5716(1) \mathrm{nm}, b=0.2864(1) \mathrm{nm}$, and $c=1.2416(2) \mathrm{nm}]$ are close to those reported in previous publications. The sample is clearly an insulator: its resistance at room temperature exceeds $10^{7} \Omega$.

Superconducting quantum interference device measurements in a magnetic field of $H=10$ Oe applied along the $c$ axis were used to determine $T_{N}=24.7(2) \mathrm{K}$ in our sample in close agreement with earlier experiments. ${ }^{24,25,27}$ The sharp-

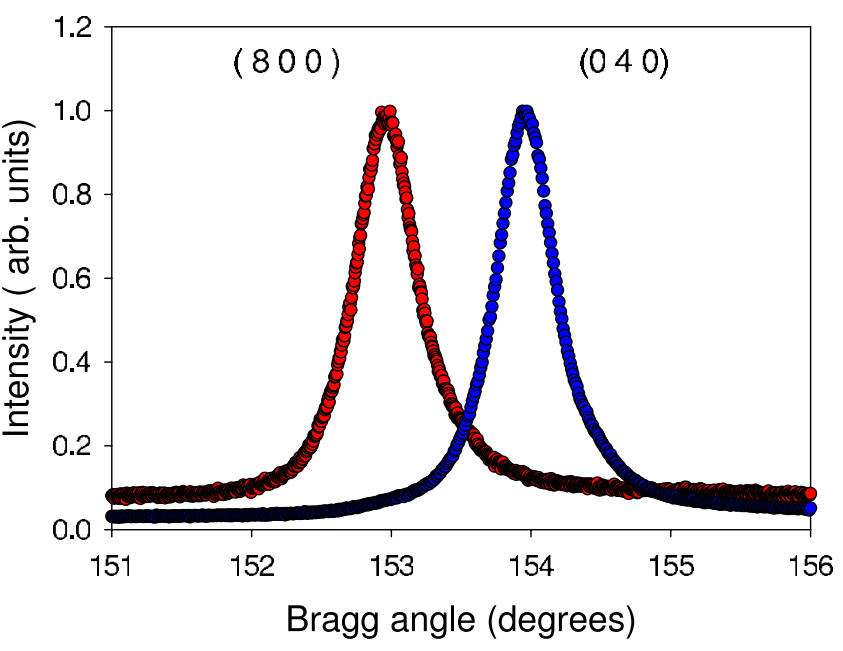

FIG. 1. (Color online) Selected reflections (left, red online: [800]; right, blue online: [040]) of XRD pattern at $T=300 \mathrm{~K}$ from two orthogonal sides of the $\mathrm{LiCu}_{2} \mathrm{O}_{2}$ crystal. Single-domain patterns are clearly seen.

ness of the transition from the AF to the PM state ensures the absence of any parasitic magnetic phases.

Time-differential $\mu^{+} S R$ measurements in zero magnetic field (ZF) with $100 \%$ polarized positive muons implanted into this single-domain $\mathrm{LiCu}_{2} \mathrm{O}_{2}$ crystal were carried out on the M20 surface muon channel at TRIUMF using the Helios spectrometer. The crystal was oriented with its $\hat{c}$ axis along the muon momentum and perpendicular to the muon polarization.

Positive muons are used as a local magnetic probe on the nanometer scale. ${ }^{29}$ Each muon stopped in the lattice (typically at an interstitial site) experiences the combined effect of external and local magnetic fields; its magnetic moment precesses at the characteristic frequency $\nu_{\mu}=\gamma_{\mu} B$, where $\gamma_{\mu} / 2 \pi=135.5388 \mathrm{MHz} / \mathrm{T}$ is the muon magnetogyric ratio and $B$ is the magnetic field at the muon site. Thus the $\mu^{+}$SR technique offers extreme sensitivity to the detection of internal magnetic fields of electronic and/or nuclear origin.

In magnetic materials, $\mu^{+} \mathrm{SR}$ measurements have long been employed to determine local magnetic fields at the muon site as this provides information on the local magnetic environment. ${ }^{29}$ One advantage of $\mu^{+}$SR over other magnetic techniques is its ability to reveal local magnetism in zero external field (ZF- $\left.\mu^{+} \mathrm{SR}\right)$ since the muons arrive into the lattice $100 \%$ polarized. Thus observation by ZF- $\mu^{+}$SR of coherent muon oscillations at a single characteristic frequency $\nu_{\mu}$ in an AF material (with long-range magnetic order) gives an unambiguous measure of the local magnetic field at the muon site. Once long-range order is lost above $T_{N}$, no coherent $\mathrm{ZF}-\mu^{+} \mathrm{SR}$ oscillation is observed. ${ }^{29}$

Observation of several different oscillatory signals is also possible in the ordered phase. This is typically taken as evidence that muons occupy several magnetically inequivalent lattice sites. However, all such signals are expected to disappear above $T_{N}$.

At low temperature, $\mathrm{ZF}-\mu^{+} \mathrm{SR}$ spectra in our $\mathrm{LiCu}_{2} \mathrm{O}_{2}$ crystal exhibit spontaneous muon spin oscillations, as expected in the ordered phase. Typical ZF- $\mu^{+}$SR spectra are shown on Fig. 2. 


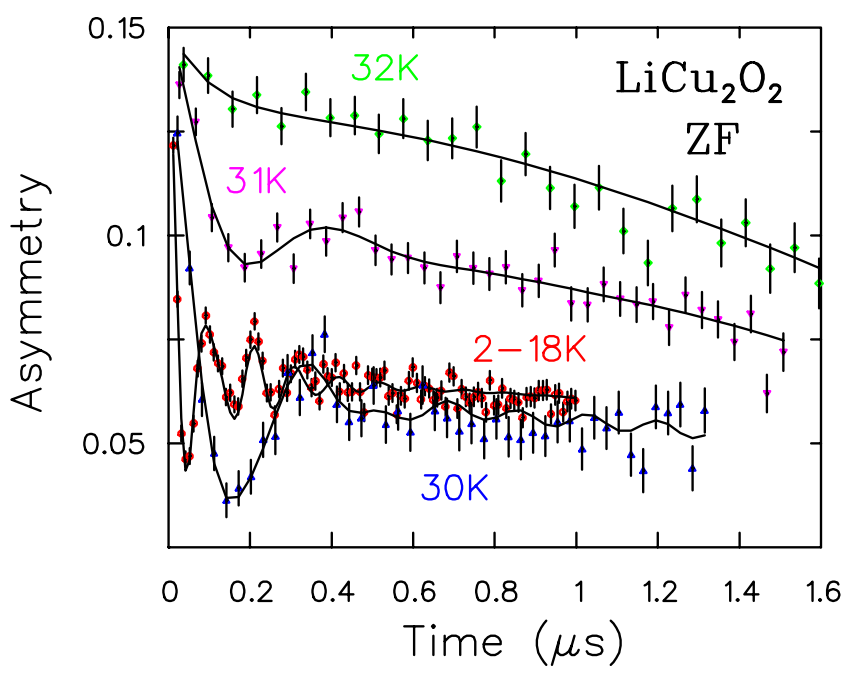

FIG. 2. (Color online) Muon spin rotation/relaxation spectra in zero magnetic field in $\mathrm{LiCu}_{2} \mathrm{O}_{2}$ crystal at different temperatures.

In this case there are two signals at different frequencies (in agreement with previous $\mu^{+} \mathrm{SR}$ measurements ${ }^{24}$ ), as shown in the inset to Fig. 3, which presents the temperature dependence of the two frequencies.

Well below $T_{N}$, our $\mu^{+}$SR spectra are similar to those in previous $\mu^{+} \mathrm{SR}$ studies. ${ }^{24}$ Accordingly, they were fit to a combination of two oscillatory and one nonoscillatory signals similar to that used by Roessli et al., ${ }^{24}$ giving an adequate description of the raw data (Fig. 2) with similar fitted parameters at low temperature. Above $T_{N}$, however, this resemblance is lost. In our experiment, the two-frequency pattern is observed up to at least $31 \mathrm{~K}$, well above $T_{N}=24.7(2) \mathrm{K}$. This discrepancy may come from the difference in quality of the samples used: experiments in Ref. 24 were carried out in a sample which consisted of "approximately ten crystals" arranged as a mosaic. X-ray studies of

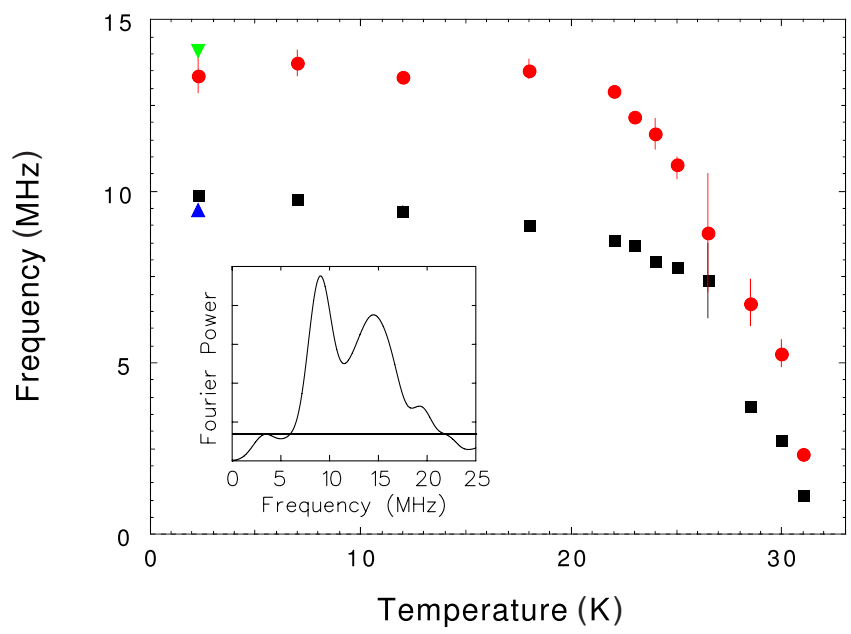

FIG. 3. (Color online) Temperature dependence of the charac-

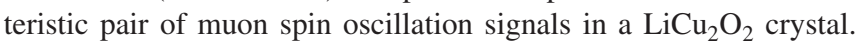
Both signals persist well above $T_{N}=24.7(2) \mathrm{K}$. Circles and squares: spectra taken with muon spin direction along the $b$ axis. Triangles: spectrum taken with muon spin direction along the $a$ axis. Inset: typical Fourier power spectrum (at $\mathrm{T}=18 \mathrm{~K}$ ). those crystals showed that they contained $\sim 3 \%$ of a parasitic $\mathrm{Li}_{2} \mathrm{CuO}_{2}$ phase. ${ }^{24}$

Based on this observation we conclude that the local magnetic environment around the muon is fundamentally different from the rest of the crystal: coherent muon spin oscillations are impossible in the disordered phase expected above $T_{N}$. As the muon creates its own local environment, it does not notice the successive phase transitions at $T_{N_{1}}=22.5 \mathrm{~K}$ and around $T_{N_{2}}=2-25 \mathrm{~K}$ detected by heat capacity measurements in $\mathrm{LiCu}_{2} \mathrm{O}_{2} \cdot{ }^{24}$

In insulators ${ }^{30,31}$ and semiconductors, ${ }^{32,33}$ the positive muon can bind an electron to form a muonium $(\mathrm{Mu})$ atom analogous to a hydrogen atom in which the proton is replaced by a muon. ${ }^{29}$ A positive muon thus acts as Coulomb attractive center for electron localization. ${ }^{34}$ In an array of magnetic ions, the long-range Coulomb interaction ensures initial electron capture while the short-range exchange interaction provides further localization into a MP bound to the muon. This phenomenon of BMP formation in a PM has recently been demonstrated in $\mathrm{SmS}$ (Ref. 5) as well as in other magnetic semiconductors. ${ }^{6}$ In an AF in the BMP thus formed, the increase in the electron kinetic energy due to localization is expected to be compensated by the on-site exchange interaction $I S / 2$ of the electron with local spin $S$ combined with Coulomb interaction with the muon as opposed to the energy $N J S^{2}$ required to flip $N$ local spins $S$ with the effective exchange energy $J$ of an AF to produce a FM "droplet" within the radius $R$ so that the change in the free energy,

$$
\Delta F=\frac{\hbar^{2}}{2 m R^{2}}-I \frac{S}{2}-\frac{e^{2}}{\varepsilon R}+N J S^{2},
$$

has a minimum as a function of $R$ - the radius of the electron's confinement. ${ }^{5,9}$

In a strong magnetic field $B$, formation of such a BMP (a $\mathrm{Mu}$ atom involving local spins) manifests itself as a twofrequency precession with both signals positioned symmetrically (except for a possible FM shift) about $\nu_{\mu}=\gamma_{\mu} B .^{5,29}$ These signals correspond to two muon spin-flip transitions between states with the same electron spin orientation (either up or down) according to the Breit-Rabi Hamiltonian. ${ }^{29}$

We deduce $B \approx 0.09 \mathrm{~T}$ from the average of the frequencies shown in Fig. 3, which is in the range of typical magnetic fields on the interstitial muon in AF materials. On the other hand, the splitting of these lines provides a measure of the muon-electron hyperfine coupling $A$, which is determined by the probability density of the electron wave function at the muon. ${ }^{29}$ In a magnetic field high enough that $\gamma_{\mu} B \gg A$, for a $\mathrm{Mu}$ atom with $A \ll A_{\mathrm{vac}}=4463 \mathrm{MHz}$ (the hyperfine coupling for a $\mathrm{Mu}$ atom in vacuum) this splitting $\Delta \nu=A$, independent of temperature, ${ }^{5,29}$ as is the case in $\mathrm{LiCu}_{2} \mathrm{O}_{2}$ below about $20 \mathrm{~K}$ (see Fig. 3). From these measurements we deduce that $A=3.5(3) \mathrm{MHz}$ for the $\mathrm{Mu}$ atom (BMP) in $\mathrm{LiCu}_{2} \mathrm{O}_{2}$. As a crude approximation, within the hydrogenlike model the hyperfine coupling scales as $1 / R^{3}$, where $R$ is the characteristic Bohr radius of the corresponding $1 s$ wave 
function. This gives $R=0.55(5) \mathrm{nm}$; the corresponding sphere is about the size of the entire unit cell of $\mathrm{LiCu}_{2} \mathrm{O}_{2}$, which include about ten magnetic $\mathrm{Cu}^{2+}$ ions.

Electron localization into a BMP in $\mathrm{LiCu}_{2} \mathrm{O}_{2}$ is ensured by the combined effects of the Coulomb and exchange interactions. The leading role of the exchange interaction is obvious from the fact that we do not see the Mu atom (BMP) at higher temperature. The increase in the electron kinetic energy due to confinement is compensated by the difference in exchange energy between the final FM and the initial AF states. ESR line broadening in $\mathrm{LiCu}_{2} \mathrm{O}_{2}$ above $T_{N}$ is associated with short-range AF correlations ${ }^{25}$ which provide a significant exchange contribution for electron localization around the muon even $6 \mathrm{~K}$ above $T_{N}$. Once these correlations are lost, the electron avoids localization, the BMP does not form, and we do not see characteristic two-frequency $\mathrm{Mu}$ precession.
As the exchange coupling is the dominant source for the electron localization and the localization radius is comparatively large in $\mathrm{LiCu}_{2} \mathrm{O}_{2}$, the role of the muon as a localization center seems to be quite modest: exchange interaction might ensure electron localization around any charged or even neutral defect in the lattice.

In conclusion, we have detected electron localization into a magnetic polaron bound to the positive muon in $\mathrm{LiCu}_{2} \mathrm{O}_{2}$. The exchange interaction, with about ten magnetic $\mathrm{Cu}^{2+}$ ions, is proposed to be the main source for electron confinement within $R \approx 0.55 \mathrm{~nm}$. This scenario of charge carrier localization might apply to other low-dimensional cuprates, including high- $T_{c}$ materials.

This work was supported by the Kurchatov Institute and the Natural Sciences and Engineering Research Council of Canada. *mussr@triumf.ca

${ }^{1}$ T. Holstein, Ann. Phys. 8, 343 (1959).

${ }^{2}$ E. L. Nagaev, Colossal Magnetoresistance and Phase Separation in Magnetic Semiconductors (Imperial College Press, London, 2002).

${ }^{3}$ P. G. de Gennes, Phys. Rev. 118, 141 (1960).

${ }^{4}$ S. von Molnár and P. A. Stampe, in Handbook of Magnetism and Advanced Magnetic Materials, edited by H. Kronmueller and S. Parkin (John Wiley \& Sons, Ltd., New York, 2007), Vol. 5.

${ }^{5}$ V. G. Storchak, J. H. Brewer, D. J. Arseneau, S. L. Stubbs, O. E. Parfenov, D. G. Eshchenko, E. Morenzoni, and T. G. Aminov, Phys. Rev. B 79, 193205 (2009).

${ }^{6}$ V. G. Storchak et al., Physica B 404, 896 (2009); 404, 899 (2009).

${ }^{7}$ L. Liu and J. T. C. Liu, Phys. Rev. B 33, 1797 (1986).

${ }^{8}$ D. Emin and T. Holstein, Phys. Rev. Lett. 36, 323 (1976).

${ }^{9}$ D. Emin and M. S. Hillery, Phys. Rev. B 37, 4060 (1988).

${ }^{10}$ E. I. Rashba, in Excitons, edited by E. I. Rashba and M. D. Sturge (Nauka, Moscow, 1985), Chap. 13, pp. 385-423.

${ }^{11}$ A. Mauger and D. L. Mills, Phys. Rev. Lett. 53, 1594 (1984).

${ }^{12}$ A. Mauger and D. L. Mills, Phys. Rev. B 31, 8024 (1985).

${ }^{13}$ P. W. Anderson, Science 235, 1196 (1987).

${ }^{14}$ V. J. Emery, Phys. Rev. Lett. 58, 2794 (1987).

${ }^{15}$ J. E. Hirsch, Phys. Rev. Lett. 59, 228 (1987).

${ }^{16}$ O. Gunnarsson and O. Rosch, J. Phys.: Condens. Matter 20, 043201 (2008).

${ }^{17}$ N. F. Mott, J. Phys.: Condens. Matter 5, 3487 (1993).

${ }^{18}$ V. J. Emery, S. A. Kivelson, and H. Q. Lin, Phys. Rev. Lett. 64, 475 (1990)

${ }^{19}$ A. L. Chernyshev and R. F. Wood, in Models and Methods of $H i g h-T_{c}$ Superconductivity: Some Frontal Aspects, edited by J.
K. Srivastava and S. M. Rao (Nova Science Publishers, Inc., Hauppauge, NY, 2003), Vol. 1.

${ }^{20}$ S. R. White and I. Affleck, Phys. Rev. B 64, 024411 (2001).

${ }^{21}$ M. Matsuda et al., Phys. Rev. B 55, R11953 (1997).

${ }^{22}$ K. M. Kojima et al., Phys. Rev. Lett. 78, 1787 (1997).

${ }^{23}$ T. Masuda, A. Zheludev, B. Roessli, A. Bush, M. Markina, and A. Vasiliev, Phys. Rev. B 72, 014405 (2005).

${ }^{24}$ B. Roessli, U. Staubb, A. Amatoc, D. Herlachc, P. Pattisond, K. Sablinae, and G. A. Petrakovskiie, Physica B 296, 306 (2001).

${ }^{25}$ S. Zvyagin, G. Cao, Y. Xin, S. McCall, T. Caldwell, W. Moulton, L. C. Brunel, A. Angerhofer, and J. E. Crow, Phys. Rev. B 66, 064424 (2002).

${ }^{26}$ M. Papagno, D. Pacile, G. Caimi, H. Berger, L. Degiorgi, and M. Grioni, Phys. Rev. B 73, 115120 (2006).

${ }^{27}$ S. Park, Y. J. Choi, C. L. Zhang, and S. W. Cheong, Phys. Rev. Lett. 98, 057601 (2007).

${ }^{28}$ S. Seki, Y. Yamasaki, M. Soda, M. Matsuura, K. Hirota, and Y. Tokura, Phys. Rev. Lett. 100, 127201 (2008).

${ }^{29}$ J. H. Brewer, in Encyclopedia of Applied Physics (VCH Publishers, New York, 1994), Vol. 11, p. 23.

${ }^{30}$ V. Storchak, J. H. Brewer, and G. D. Morris, Phys. Rev. Lett. 75, 2384 (1995).

${ }^{31}$ V. G. Storchak, D. G. Eshchenko, J. H. Brewer, G. D. Morris, S. P. Cottrell, and S. F. J. Cox, Phys. Rev. Lett. 85, 166 (2000).

${ }^{32}$ V. Storchak, S. F. J. Cox, S. P. Cottrell, J. H. Brewer, G. D. Morris, D. J. Arseneau, and B. Hitti, Phys. Rev. Lett. 78, 2835 (1997).

${ }^{33}$ D. G. Eshchenko, V. G. Storchak, J. H. Brewer, and R. L. Lichti, Phys. Rev. Lett. 89, 226601 (2002).

${ }^{34}$ V. G. Storchak, D. G. Eshchenko, and J. H. Brewer, J. Phys.: Condens. Matter 16, S4761 (2004). 